\title{
Endoscopic versus conventional septoplasty in the treatment of obstructive nasal septal deviation
}

\author{
Salah Nassrallah', Mircea Cristian Neagos ${ }^{2}$, Gheorghe Muhlfay ${ }^{3}$, Adriana Neagos ${ }^{3}$ \\ "George Emil Palade" University of Medicine, Pharmacy, Science and Technology, Targu Mures, Romania \\ 2"Carol Davila" University of Medicine and Pharmacy, Bucharest, Romania \\ ${ }^{3}$ ENT Department, "George Emil Palade" University of Medicine, Pharmacy, Science and Technology, \\ Targu Mures, Romania
}

\begin{abstract}
BACKGROUND. Nasal septum deviations are disorders in which the nasal septum has an abnormal conformation that affects nasal breathing. Surgical correction of the deviated nasal septum, performed by various techniques, is one of the methods of repermeabilization of the upper airway. The aim of the present study was to evaluate the efficacy of endoscopic surgical treatment of the nasal septum deviation on the nasal obstruction, as well as to evaluate the frequency of postoperative complications compared to classical septoplasty. MATERIAL AND METHODS. A retrospective longitudinal study was performed on a group of 92 patients diagnosed with nasal septal deviation, between 2014-2018. 32 patients were excluded from the study after the exclusion criteria were applied. Two groups of 30 patients each were formed: Group I, endoscopic septoplasty was performed; Group II, the conventional septoplasty was used. An objective endoscopic and rhinomanometric evaluation, as well as a subjective assessment of symptoms (nasal obstruction, headache, posterior rhinorrhea, sneezing), was performed preoperatively and at 3 months postoperatively.
\end{abstract}

RESULTS. The study showed better results $(\mathrm{p}<0.05)$ and fewer complications in endoscopic septoplasty compared to the traditional one, the endoscopic septoplasty providing better lighting and improved access, allowing a limited incision.

CONCLUSION. Endoscopic septoplasty can be considered a reliable alternative to traditional techniques. It is essential to correctly identify the type of preoperative deformity in order to select the appropriate surgical strategy.

KEYWORDS: nasal septal deviation, endoscopic septoplasty, nasal endoscopy, nasal obstruction.

\section{INTRODUCTION}

The respiratory system consists of the upper and lower respiratory tracts, lungs, as the main organs of the respiratory act. The nostrils are the starting point of physiological nasal breathing, initiated with the inspiration of oxygen-rich atmospheric air and completed by the expiration of air from the lungs, rich in $\mathrm{CO}_{2}{ }^{1}$.

The role of the nasal fossae, through the nasal mucosa, is to condition the inspired air through the following three processes: heating, humidification and filtration. A qualitatively modified air will reach the pharynx, which will protect the mucosa of the respiratory tract through its physical parameters. For the proper development of these processes it is necessary the presence of a sufficient respiratory flow through the nostrils and a continuous mucus layer on the surface of their mucosa. The whole conditioning process is selfregulated depending on the parameters of the inspired air and the body's momentary respiratory needs.

The calibre of the nasal fossa, through the architecture and the morpho-functional integrity of the mucosa, through its vasculo-erectile function, contributes to the regulation of the respiratory air flow.

The nasal fossa and the paranasal sinuses also have a role of resonant cavity, being able to modify the quality of the voice through the changes of volume and permeability. The vocal timbre may change in relation to the size of the nostrils and 


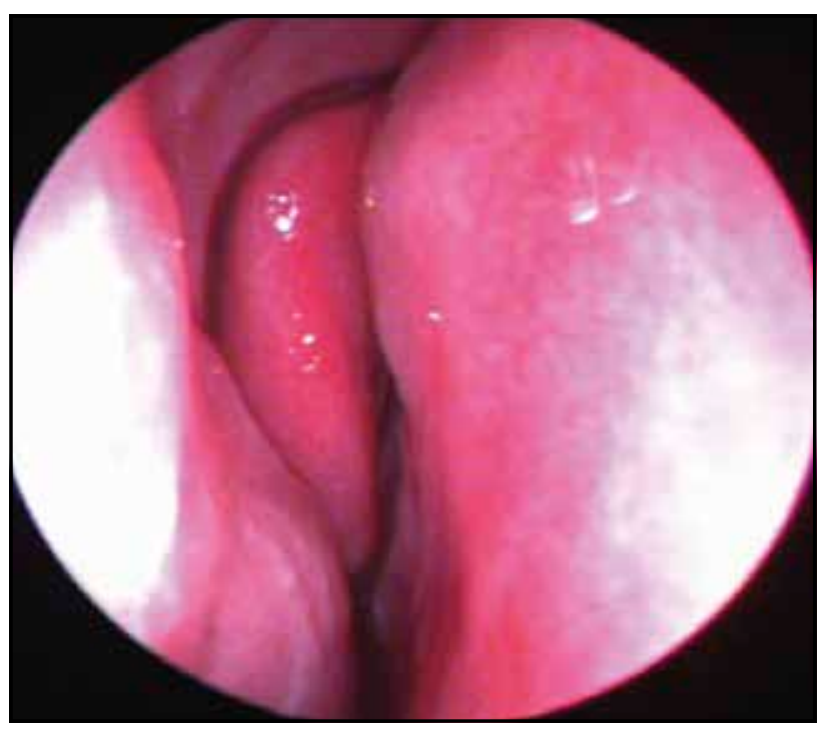

Figure 1. Nasal endoscopy image - nasal septal deviation with compressive effect on the middle nasal turbinate.

closed rhinolalia may occur in the case of nasal obstruction, or open rhinolalia if a much larger air column passes through the nasal passages.

Excitation of receptors (thermal, tactile, olfactory) in the nasal mucosa informs the superior nervous centres and the cerebral cortex, which reflexively maintain the respiratory functional balance. The nasal mucosa, by excitation of the trigeminal endings, reflexively causes changes in pulmonary ventilation in terms of rhythm, frequency and amplitude.

The upper airways are responsible for a major component of the total airway resistance; they provide $40-70 \%$ of total lung resistance during resting breathing. The size and collapsibility of the upper airway vary dynamically during the respiratory cycle and is also affected by the respiratory tract - oral or nasal, lung volume, ventilation level, hypoxia, hypercapnia. In addition, upper airway resistance can increase the effort of inspiratory muscles to maintain a normal airflow ${ }^{2-4}$.

Clinical studies have shown that the pressure-flow relationship in the airways falls within the Starling resistor theory, which involves blocking the upper airways when the upstream and downstream pressure remains below a critical value. The flow limitation will result from the linear increase in airflow, so that the upstream pressure is increased above the critical pressure ${ }^{3,5}$.

Nasal obstruction can significantly influence all this described mechanism, has a multifactorial etiology and involves the existence of inflammatory, infectious, tumor processes or structural abnormalities. The most common causes of nasal obstruction include the nasal septum deviation and hypertrophy of the inferior nasal turbinates.
Nasal septum deviations are disorders in which the nasal septum has an abnormal conformation that affects nasal breathing. Intranasal deformities are often asymptomatic, which is why only $19 \%$ of patients who have mouth breathing, secondary to poor nasal breathing, are aware of the nasal respiratory disorder. The rest of the patients present adjacent symptoms: breathing difficulty with sleep disorders, dryness of the nasal mucosa and sometimes facial pain ${ }^{6,7}$.

There are several clinical classifications of nasal septal deviations, but the most common used in everyday practice is the following: type I - a slight deviation in the vertical or horizontal plane that does not extend over the entire vertical portion of the septum, type II - anterior vertical deviation, type III - posterior vertical deviation, type IV - "S-shaped" septum, type V horizontal deviation on one side with or without significant deformities on the opposite side, type VI a type V with a groove on the concave side, type VII - any combination of types II - VI. Mladina divided the septal deviations into two main groups: the socalled vertical deformations (types 1,2,3 and 4) and the horizontal ones (types 5 and 6$)^{8}$. Vertical deformations have a longer axis in the vertical plane, as a result of the force acting against the nasal septum in an antero-posterior direction. Nevertheless, horizontal deformations have a longer axis in the horizontal plane, when the force against the septum acted from the upper to the lower part ${ }^{8}$. It is estimated that $80 \%$ of the population has a misalignment of the nasal septum (Figure 1).

Medical treatment of nasal obstruction consists in the administration of nasal decongestants, antihistamines, sprays with intranasal corticosteroids, in order to reduce mucosal edema, but this is only a temporary solution.

Surgical correction of the deviated nasal septum, performed by various techniques, is one of the methods of repermeabilization of the upper airway.

The aim of the present study was to evaluate the efficacy of endoscopic surgical treatment of the nasal septum deviation on the nasal obstruction, as well as to evaluate the frequency of postoperative complications compared to conventional septoplasty.

\section{MATERIAL AND METHODS}

Within the ENT Department of the Targu Mures County Emergency Clinical Hospital, a retrospective longitudinal study was performed on a group of 92 patients diagnosed with nasal septal deviation (out of a total of 690 patients with rhinosinusal pathology), in the period 2014-2018. The study was based on collecting data from the Clinic's casuistry, as well as on preoperative and postoperative evaluation of patients with nasal pathology. Thus, in order to perform surgery, patients were evalu- 

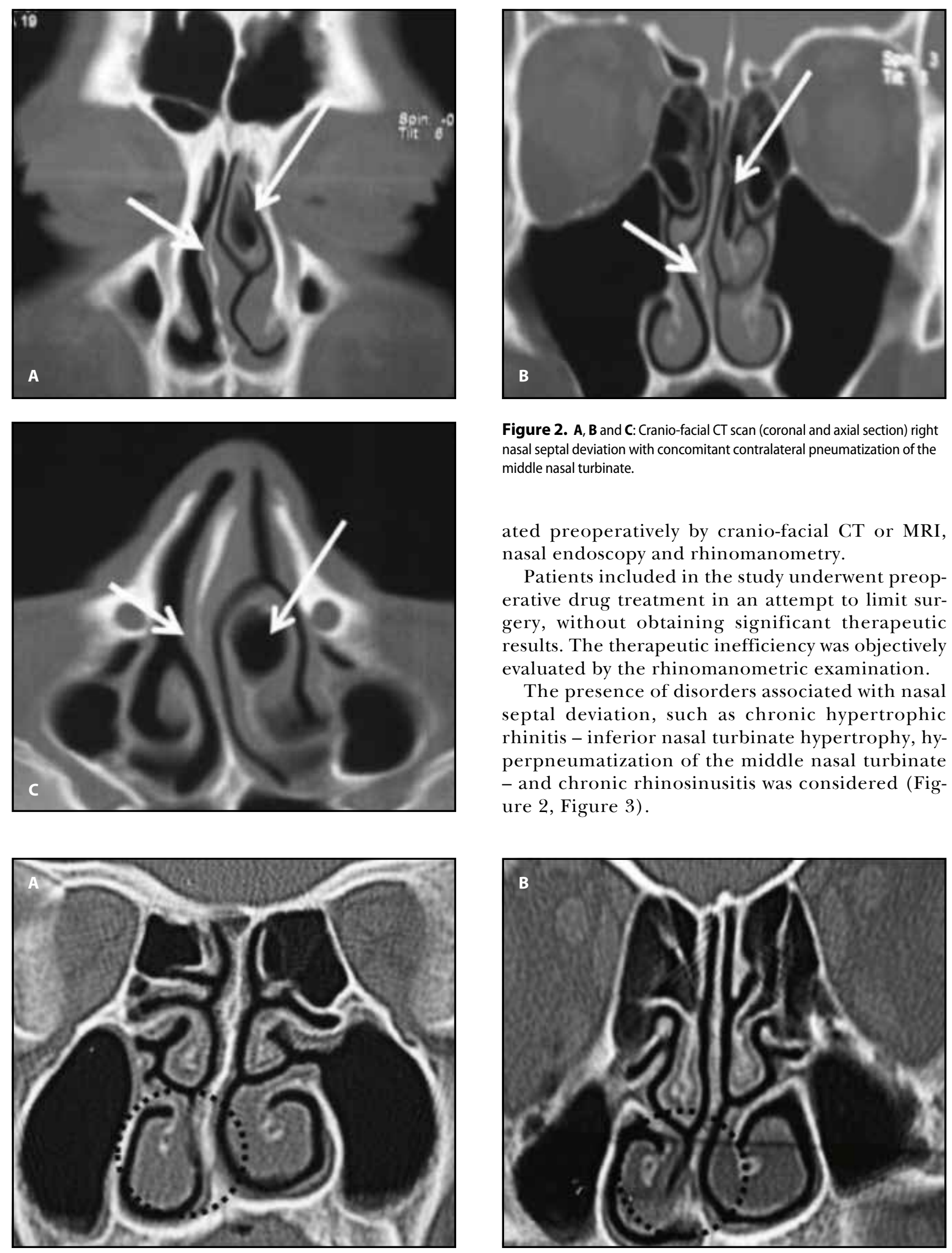

Figure 3. A and B: Cranio-facial CT scan (coronal section) - right nasal septal deviation with vertical lamellar pneumatization of the left contralateral inferior nasal turbinate.

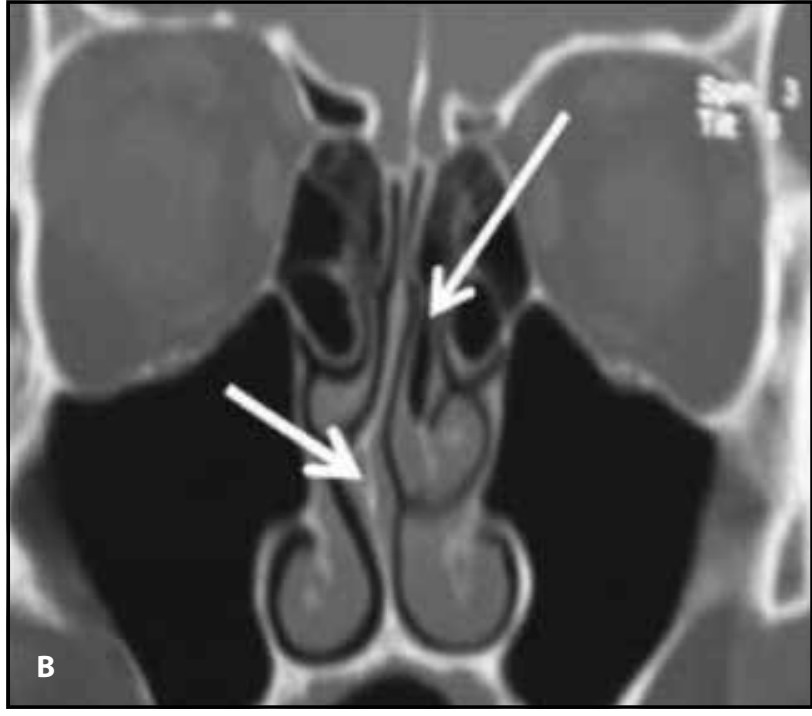

Figure 2. A, B and C: Cranio-facial CT scan (coronal and axial section) right nasal septal deviation with concomitant contralateral pneumatization of the middle nasal turbinate.

ated preoperatively by cranio-facial CT or MRI, nasal endoscopy and rhinomanometry.

Patients included in the study underwent preoperative drug treatment in an attempt to limit surgery, without obtaining significant therapeutic results. The therapeutic inefficiency was objectively evaluated by the rhinomanometric examination.

The presence of disorders associated with nasal septal deviation, such as chronic hypertrophic rhinitis - inferior nasal turbinate hypertrophy, hyperpneumatization of the middle nasal turbinate - and chronic rhinosinusitis was considered (Figure 2, Figure 3). 
Table 1. Distribution by years of nasal septum deviations operated during 2014-2018.

\begin{tabular}{ccc}
\hline $\begin{array}{c}\text { Year of } \\
\text { hospitalization }\end{array}$ & $\begin{array}{c}\text { Number of patients } \\
\text { with rhinosinusal disorders }\end{array}$ & $\begin{array}{c}\text { Number of patients with nasal septum } \\
\text { deviation (percentage ratio) }\end{array}$ \\
\hline 2014 & 119 & $28(23.5 \%)$ \\
\hline 2015 & 158 & $21(13.39 \%)$ \\
\hline 2016 & 238 & $22(9.24 \%)$ \\
\hline 2017 & 98 & $11(11.22 \%)$ \\
\hline 2018 & 77 & $10(12.98 \%)$ \\
\hline
\end{tabular}

The data were collected from: anamnesis, ENT clinical examination, nasal endoscopic examination, general clinical examination, biological and imaging examinations, histopathological results, type of intervention applied and associated treatments.

At patients' admission, the symptomatology was monitored and it was represented by: nasal obstruction and posterior rhinorrhea, along with headache and sneezing. This assessment was made subjectively according to the patients' report.

In order to establish the degree of nasal obstruction and to select patients for septoplasty, rhinomanometric measurements were performed to assess nasal flow and nasal resistance.

The analysis and storage of the collected clinical and demographic information was performed in an Excel database, and the processing of the results was done using descriptive statistics (frequencies, percentages). The relative frequencies of the variables recorded by the study were determined; correlations were made between: pathological history, recurrences. Other variables studied were: date of admission, sex, age, environment of origin, clinical manifestations, diagnostic method, treatment method, treatment outcome.

\section{RESULTS}

In the ENT casuistry of the Targu Mures County Emergency Clinical Hospital between 2014 and 2018, a total number of 690 patients with rhinosinusal pathology of different etiologies were evaluated. Of these, in a number of 92 patients (13.33\%) hospitalization was decided to treat the nasal obstruction caused by the existence of nasal septum deviations of varying degrees.

Analysing the variation of the number of cases of nasal septum deviation associated with rhinosinusal pathologies, reported by calendar years, the existence of a peak at the level of 2014 can be noticed. A decrease of the number of cases of septal deviation can be observed

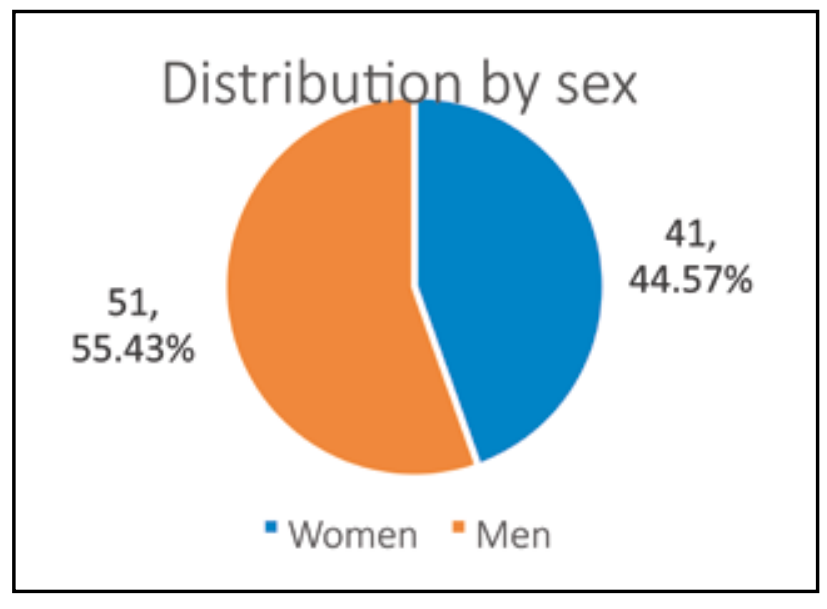

Figure 4. Distribution of nasal septal deviations by sex.

during the reported time period, as well as of the associated chronic rhinosinusitis pathology (Table 1).

Studying age groups by sex, a peak of nasal pathology (such as the deviation of the nasal septum) can be observed in the age group between 41-50 years. One of the explanations can be given by the increased incidence at this age and of the associated sinus pathology, for which patients turn to the otorhinolaryngology department. There is also a predominance of this type of pathology in male patients, possibly due to repeated aggression in the nasal area (Figure 4).

At the hospitalization of patients, the clinical examination was preceded by an anamnesis, which subjectively followed the presence of the following symptoms: nasal obstruction, posterior rhinorrhea, headache, sneezing; we considered the presence of these clinical symptoms to be associated with the presence of nasal septal deviations associated with collateral pathology such as: inferior nasal turbinate hypertrophy (IT), hyperpneumatization of the middle turbinate (MT), chronic rhinosinusitis. Evaluating the initial symptoms, it could be observed that nasal obstruction was present in a pro- 


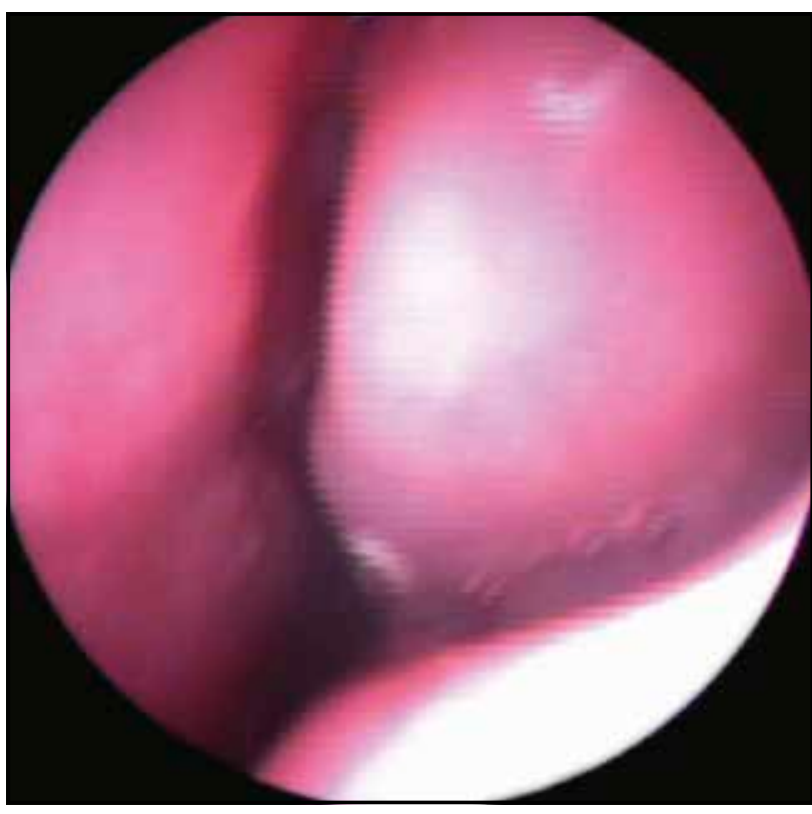

Figure 5. Endoscopic aspect of pneumatization of the middle nasal turbinate - Concha bullosa - and hypertrophy of the inferior nasal turbinate.

portion of $88.3 \%$, and posterior rhinorrhea in $75 \%$, these being clinically specific signs of nasal septal deviations with or without associated chronic rhinitis. Headache was present in $40 \%$ of patients and sneezing in $26.7 \%$ of patients, these being more specific to a rhinosinusal pathology with or without associated allergic manifestations.

The nasal endoscopic examination allowed the morphological evaluation of the nasal septum and the inferior nasal turbinates: the presence of the hypertrophy of the inferior nasal turbinates, the hyperpneumatization of the middle nasal turbinates. In our study, we found the presence of hypertrophy of the inferior nasal turbinate in a percentage of $83.33 \%$ and the hyperpneumatization of the middle nasal turbinate in a percentage of $51.67 \%$ (the evaluation was made in the group of 92 patients with nasal septum deviation) (Figure 5)

In order to evaluate the resistance of the upper airways, the anterior rhinomanometry was used, which measures both the flow and the nasal resistance, objectively establishing the degree of nasal obstruction. As active rhinomanometry is easier to perform, it is recommended in most cases. Anterior rhinomanometry may be influenced by nasal cycles as well as deformities of the nostrils. The modern standardized technique, the 4-phase or high-resolution rhinomanometry, studies ascending and descending curves separately in inspiration and expiration (Figure 6).

Nasal obstruction can be quantified as total or unilateral resistance, on each nostril separately. Thus, the resistance is related to a certain flow or pressure.

According to the results, it was possible to observe for the group of patients with nasal septal deviation an average value of nasal flow of $603.9 \mathrm{ml} / \mathrm{sec}$ with a median around $611 \mathrm{ml} / \mathrm{sec}$. These values correspond to a severe nasal obstruction, for which surgical treatment has been indicated. As a result of rhinomanometric measurements, establishing the degree of nasal obstruction, various therapeutic procedures were established.

Out of a total of 92 patients, after clinical and rhinomanometric evaluation, 32 patients were excluded due

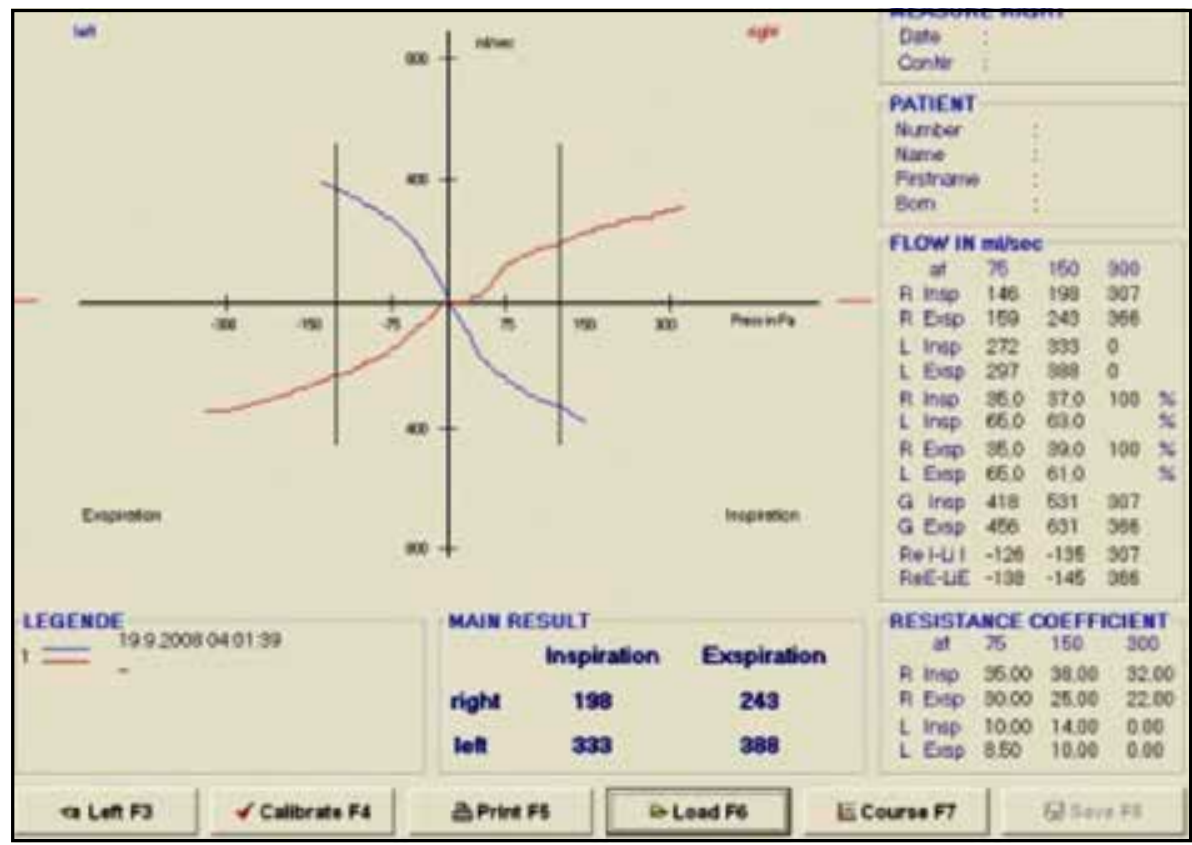

Figure 6. Rhinomanometry with highlighting the values of nasal flow and nasal resistance to certain pressure values. 
to: the presence of a moderate or mild nasal obstruction, which determined the patient's refusal to undergo surgical treatment; contraindication for general anaesthesia. In this context, the group that underwent surgery was reduced to a number of 60 patients.

On the group of 60 patients included in the study were applied, selectively, the well-known classical surgical techniques of septoplasty (Killian and Cottle), as well as procedures derived and adapted to the situations, these being represented by minimal resections of the deviated parts of the nasal septum.

The surgical interventions were practiced through two approaches: the classical septoplasty technique (Killian and / or Cottle procedure); Cottle endoscopic septoplasty. At the same time, in some cases, partial or total inferior mucotomies were performed, ablation under endoscopic guidance of the accompanying polyps, antrostomy with restoration of ostial patency where nasal pathology was associated with maxillary rhinosinusitis. These types of interventions were not quantified for the present study.

Two groups of 30 patients each were formed. In Group I, endoscopic septoplasty was performed, while in patients included in Group II, the classical septoplasty was used. Certain criteria for selecting patients for endoscopic or classical septoplasty were not specifically applied.

An objective endoscopic and rhinomanometric evaluation, as well as a subjective assessment of symptoms, was performed at 3 months postoperatively. The following parameters were monitored in both patients' groups: subjective (nasal obstruction, headache, posterior rhinorrhea, sneezing) and objective (persistence of anterior or posterior septal deviation, persistence of the septal ridge, synechiae; by nasal endoscopy).

Postoperatively, rhinomanometry was performed to objectively assess nasal obstruction, observing an increase in nasal flow up to $700 \mathrm{ml} / \mathrm{sec}$, turning a severe nasal obstruction into a mild nasal obstruction. The results were similar for both surgical techniques applied.

Nasal obstruction was also quantified subjectively. By analysing the data obtained through the subjective evaluation, we observed that the endoscopic technique allowed the reduction of postoperative nasal obstruction in 27 patients in the group of $30(90 \%)$, compared to 16 patients in the group to which the classical technique was applied (53.33\%).

Headache was reduced in 14 patients in Group I (46.67\%), compared to 10 in study Group II (33.33\%); posterior rhinorrhea was reduced in 24 of 30 patients who underwent the endoscopic technique $(80 \%)$, unlike 21 who underwent the classical technique $(70 \%)$. Sneezing is maintained postoperatively in 9 out of 30 patients in Group I (30\%) and in 7 out of 30 patients in Group II (23.33\%) (Figure 7).

Making an objective endoscopic evaluation of the shape of the postoperative nasal septum, after applying the two surgical techniques, the following can be observed. Regarding the objective postoperative evaluation, at 3 months postoperatively, the persistence of the anterior septal deviation was similar in both groups, with an inclination towards conventional septoplasty. The persistence of posterior septal deviation was less common in endoscopic septoplasty. Residues of the septal ridge were observed postoperatively only after classical septoplasty, as in the case of postoperative synechiae (Table 2). The results of the endoscopic evaluation show us a statistically significant efficacy of endoscopic septoplasty compared to the conventional one $(\mathrm{p}<0.05)$.

\section{DISCUSSIONS}

Deviation of the nasal septum is a common structural cause of nasal obstruction and can occur

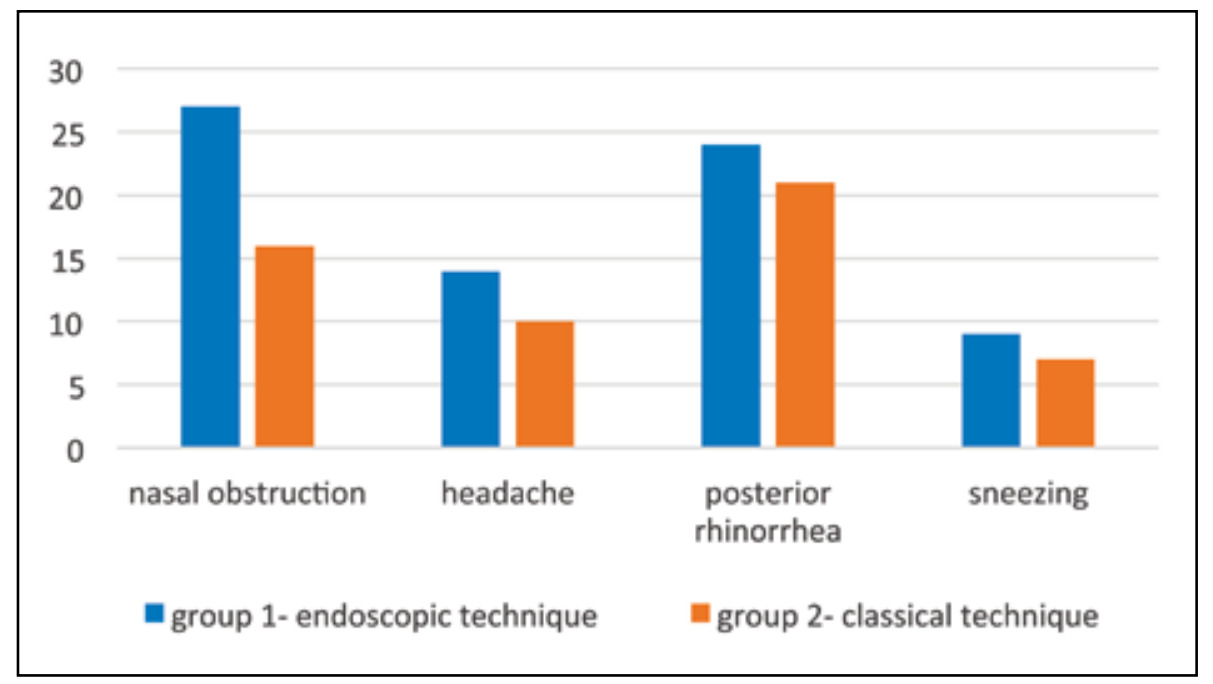

Figure 6. Postoperative comparative data obtained based on the evaluation of the endoscopic technique vs. the conventional septoplasty technique. 
Table 2. Postoperative evaluation 3 months after surgery - endoscopic vs. conventional techniques.

\begin{tabular}{cccc}
\hline Objective evaluation at $\mathbf{3}$ months & Conventional septoplasty $\mathbf{N}=\mathbf{3 0}$ & Endoscopic septoplasty $\mathbf{N}=\mathbf{3 0}$ & The value of $\mathbf{p}$ \\
\hline Persistence of anterior septal deviation & $10 \%$ & $6.7 \%$ & $>0.05$ \\
\hline Persistence of posterior septal deviation & $23.3 \%$ & $10 \%$ & $<0.05$ \\
\hline Persistence of the septal ridge & $13.3 \%$ & $0 \%$ & $<0.05$ \\
\hline Septo-turbinal synechiae & $36.7 \%$ & $0 \%$ & $<0.05$ \\
\hline
\end{tabular}

from dislocation of the quadrangular cartilage from its borders in its bony quadrant or from an intrinsic deformity affecting the vomer, the perpendicular plate of the ethmoid and / or the quadrangular cartilage itself. It can be found at any age, with a prevalence of $1.45 \%-6.3 \%$. The etiology of nasal septal deviation can be classified as congenital, with genetic effects that cause aberrant growth, post-traumatic and infectious or even mass effect in the case of neoplasms of the nasal cavity.

Nasal septal deviation occurs more frequently in the male population, with a male-female ratio of $1.72: 1$, and the most affected age groups are those in the second and third decades of life, according to the results of our study. The incidence of interventions at these ages can be explained by several theories. In children, during the intervention, the germination centres of growth can be damaged and it would lead to the underdevelopment of the nose; and indications would be reserved only for cases of bilateral nasal obstruction. In adults over the age of 60 , a process of calcification of the septum can occur with an increased risk of septal fracture and its spread to the base of the skull.

In the present study, the most common symptoms of patients with nasal septal deviation are nasal obstruction $(88.33 \%)$, posterior rhinorrhea $(75 \%)$, headache $(40 \%)$ and sneezing $(26.7 \%)$. The frequency of symptoms of nasal obstruction (75\%) and rhinorrhea (55\%) were similar to those of the study carried out by Gupta et al. (2005), but headache was the major secondary symptom in their study9.

Nathan et al. (2002) found that the pathology of the lateral nasal wall is associated with the deviation of the nasal septum, the most common and most important being inferior turbinate hypertrophy $(75 \%)$, followed by pneumatization of the middle turbinate ${ }^{10}$. In our study, we found the presence of inferior turbinate hypertrophy in a percentage of $83.33 \%$ and hyperpneumatization of the middle turbinate in a percentage of $51.67 \%$.

The concept of septoplasty was first popularized by Killian (1904) and Freer (1902) more than 100 years ago, and in 1947, Cottle defined surgical septoplasty as a treatment for correcting nasal airway obstruction and standardized the technique.

The application of endoscopic techniques to correct septal deformities was first described in 1991 by Stammberger. Ever since, endoscopic septoplasty has been used not only to treat symptomatic nasal obstruction, but also to improve surgical access to the middle meatus, the technique being used as an adjunct in endoscopic surgery of the paranasal sinuses ${ }^{10,11}$.

An ideal surgical correction of the nasal septum should meet the following criteria: remove nasal obstruction; be conservative; not to produce iatrogenic deformations; not to compromise the osteomeatal complex; to be able to carry out a revision intervention if necessary; to preserve the functions of the nose. In the present study, no complications were observed in the group in which endoscopic septoplasty was performed, while in the study of Banglawala et al. ${ }^{12}$, the percentage of complications was $2.08 \%$, respectively $5 \%$ in the study conducted by Kim et al. ${ }^{13}$.

Although classical septoplasty improves nasal breathing, most of the time, it does not meet all the above criteria. This is due to poor visualization, difficult accessibility, poor lighting, and / or difficulty in correctly assessing the pathology that may predispose to mucosal damage and that may compromise endoscopic visualization during sinus surgery. Unnecessary resection or manipulation with overexposure of the septal frame may reduce the possibility of a revision, especially when treating a narrow nasal cavity.

According to Kim et al., endoscopic septoplasty has several advantages in addition to the classical technique, since it can be used in the surgical treatment of allergic diseases ${ }^{13}$. It can also be considered as an effective learning method. In fact, the live broadcast of the procedure on a monitor can be an opportunity to record and study the anatomy, pathology and surgical technique by surgeons attending the intervention, by specialist doctors or by medical students.

The endoscopic technique provides significantly better visibility of the working field, especially in posterior septal deviations. The endoscope can be easily inserted under the septal mucosa with minimal lift to allow excellent viewing. The incision can be made more posteri- 

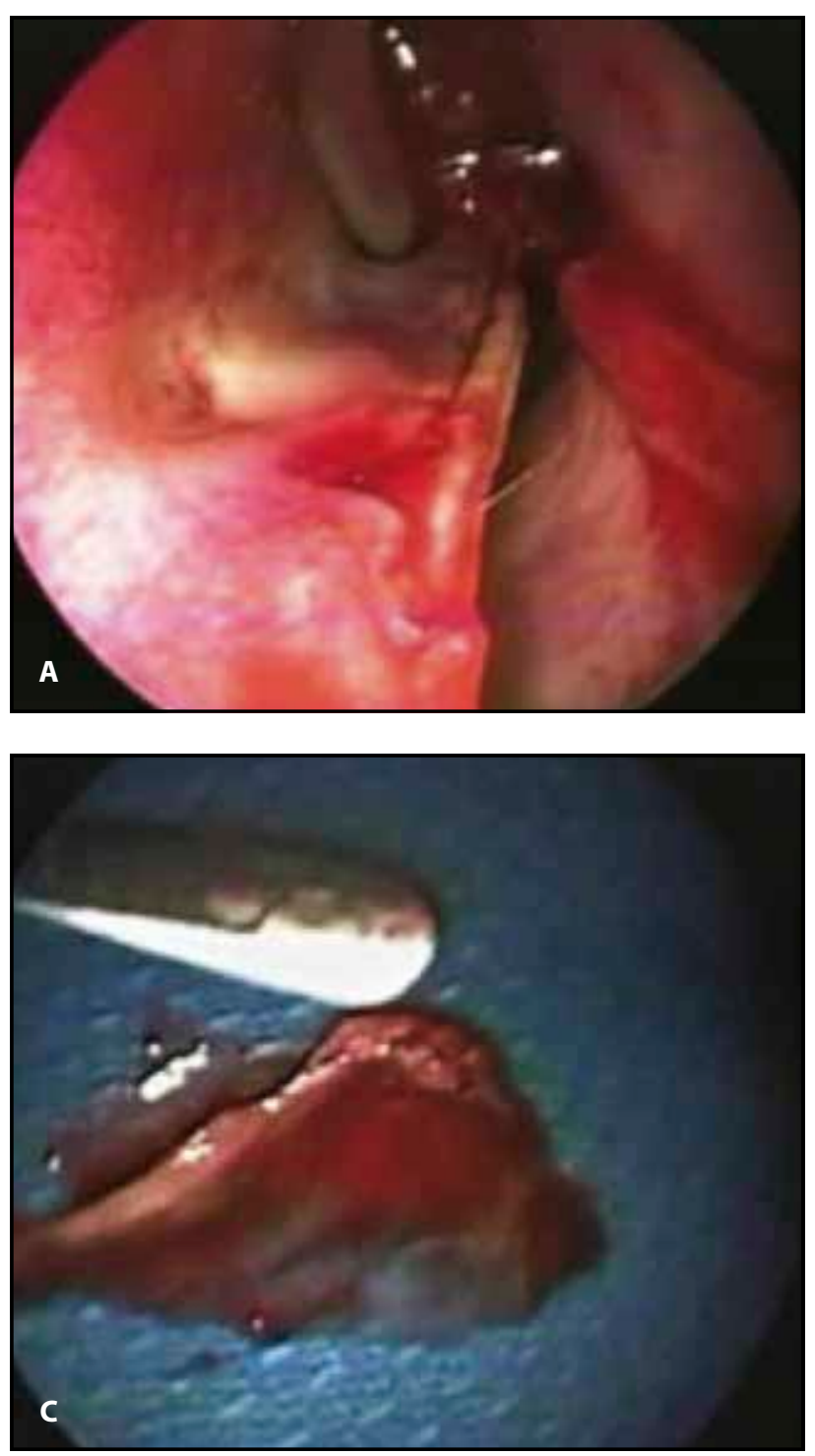

orly, inside the nose, in the immediate vicinity of the deviation, the anterior surface of the detached septal mucosa is significantly reduced, thus having an important advantage in limiting postoperative edema.

After performing this study and analysing the data obtained, it is found that endoscopic septoplasty reduces the contact area by allowing intraoperative evaluation. It is associated with a marked reduction in patient morbidity, both preoperatively and postoperatively.

The endoscopic septoplasty technique has long-term advantages, a clear example being revision surgery, when the septal mucosa is often adherent due to submucoperichondrial dissection and cartilaginous resection, when conventional surgical techniques are applied. Detachment in these areas is difficult, in the best case, and can affect the integrity of the mucosa. The incision made in the immediate vicinity of the remaining septal deviation, under endoscopic control, and the detachment of the mucosa without underlying

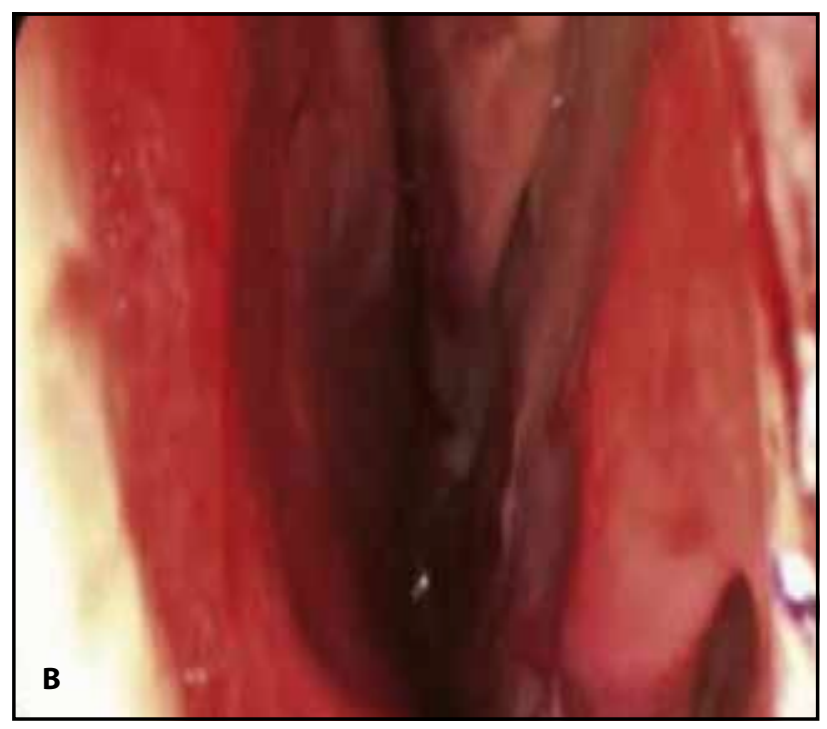

Figure 8. (A, B and C): Pre- and postoperative endoscopic evaluation of the left septal ridge.

cartilage is thus minimized or eliminated. This is an essential advantage in patients with previous operations for deviation of the nasal septum.

However, there are limitations of the endoscopic surgical technique, which includes the loss of binocular vision, the need for frequent cleaning of the tip of the endoscope, especially in case of bleeding, and last but not least, the inability to correct under endoscopic control the complex deformities of the nasal septum with caudal deviations (Figure 8).

A significantly high rate of reduction of postoperative symptoms was observed in the group of patients operated by endoscopic technique, compared to the classical technique, results that are similar to those of the study conducted by Nathan et al. ${ }^{11}$.

Sarfaraz and Young Hyo Kim et al..$^{13,14}$ observed a significant improvement in nasal obstruction and headache in the group that underwent endoscopic septoplasty, also expressing the importance of minimal post-septoplasty nasal packing in patients' subsequent evolution.

Paradis et al., in a study performed on 63 patients operated classically and endoscopically for septal deviation, conclude that there were subjective improvements in nasal obstructive symptoms in both groups, but no significant differences between endoscopic and classical septoplasty ${ }^{15}$. Paradis et al. ${ }^{15}$ observed that postoperative synechiae were formed in significantly smaller numbers in patients in the group with endoscopic septoplasty than in the group with classical septoplasty, similar data being observed in our study.

In the present study, a higher number of patients showed a decrease in nasal symptoms in general, on all levels, after performing endoscopic septoplasty compared to the second group, as shown by all the statisti- 
cal data listed above. The studies of Tehnia Aziz et al. from 2014 as well as the studies of G. Dell'Aversana Orabona et al. from 2018 complete, by measurements of the parameters as well as by the nasal evaluation after endoscopic septoplasty of the septal deformities, the results of our study according to the specialized literature ${ }^{16,17}$.

\section{CONCLUSIONS}

Although endoscopic septoplasty can be considered a reliable alternative to traditional techniques, it is essential to correctly identify the type of preoperative deformity in order to select the appropriate surgical strategy. Long-term follow-up and larger series of patients is a necessity to more accurately assess the indications and limitations of endoscopy-assisted septoplasty in all cases and types of septal deviation.

The study showed better results and fewer complications in endoscopic septoplasty compared to the traditional one, the endoscopic septoplasty providing better lighting and improved access, allowing a limited incision. This technique causes less trauma at the septal level, thus reducing postoperative complications.

Conflict of interests: The authors declare that they have no conflict of interest

Authors contribution: Nassrallah Salah and Neagos Adriana read, write and approved the final manuscript; Neagos Cristian Mircea has contribution to the statistics, and Muhlfay Gheorghe approved the final manuscript; Neagos Adriana is the corresponding author too.

\section{REFERENCES}

1. Teixeira J, Certal V, Chang ET, Camacho M.. Nasal septal deviations: a systematic review of classification systems. Plast Surg Int. 2016;2016:7089123. DOI: 10.1155/2016/7089123.

2. Stumpe MR, Chandra RK. Disorders of the nasal septum. In: Stucker FJ, de Souza C, Kenyon GS, Lian TS, Draf W, Schick B. Rhinology and Facial Plastic Surgery. Springer Berlin Heidelberg; 2009, p.151-63.
3. Solyom R, Csiszer I, Neagos A. Tonsillar hypertrophy implications in sleep disorders in adults and children. Rom J Morphol Embriol. 2014;55(2 Suppl):603-6.

4. Kumar L, Belaldavar BP, Bannur H. Influence of deviated nasal septum on nasal epithelium: an analysis. Head Neck Pathol. 2017;11(4):501-5. DOI: 10.1007/s12105-017-0819-9.

5. Nickels FA. Diseases of the nasal cavity. Vet Clin North Am Equine Pract. 1993;9(1):111-21. DOI: 10.1016/S0749-0739(17)30418-2.

6. Tan HM, Chong VFH. CT of the paranasal sinuses: Normal anatomy, variants and pathology. CME Radiology. 2001;2(3):120-5.

7. Jones N. The nose and paranasal sinuses physiology and anatomy. Adv Drug Deliv Rev. 2001;51 (1-3):5-19. DOI: 10.1016/s0169-409x(01)00172-7.

8. Mladina R, Skitarelić N, Poje G, Šubarić M. Clinical implications of nasal septal deformities. Balkan Med J. 2015;32(2):137-46. DOI: 10.5152/balkanmedj.2015.159957.

9. Gupta M, Motwani G. Comparative study of endoscopic aided septoplasty and traditional septoplasty in posterior nasal septal deviations. Indian J Otolaryngol Head Neck Surg. 2005;57(4):309-11. DOI: 10.1007/ BF02907695.

10. Sautter NB, Smith TL. Endoscopic septoplasty. Otolaryngol Clin North Am. 2009;42(2):253-60. DOI: 10.1016/j.otc.2009.01.010.

11. Lund VJ, Holmstrom M, Scadding GK. Functional endoscopic sinus surgery in the management of chronic rhinosinusitis. An objective assessment. J Laryngol Otol. 1991;105(10):832-5. DOI: 10.1017/ s0022215100117643.

12. Banglawala SM, Gill M, Sommer DD, Psaltis A, Schlosser R, Gupta M. Is nasal packing necessary after septoplasty? A meta-analysis. Int Forum Allergy Rhinol. 2013;3(5):418-24. DOI: 10.1002/alr.21110.

13. Kim J, Kim SW, Kim SW, Cho JH, Park YJ. Role of the sphenoidal process of the septal cartilage in the development of septal deviation. Otolaryngol Head Neck Surg. 2012;146(1):151-5. DOI: 10.1177/0194599811425000.

14. Kim YH, Kim BJ, Bang KH, Hwang Y, Jang TY. Septoplasty improves life quality related to allergy in patients with septal deviation and allergic rhinitis. Otolaryngol Head Neck Surg. 2011;145(6):910-4. DOI $10.1177 / 0194599811424119$.

15. Paradis J, Rotenberg BW. Open versus endoscopic septoplasty: a single-blinded, randomized, controlled trial. J Otolaryngol Head Neck Surg. 2011;40(Suppl 1):S28-33. Effectiveness of endoscopic septoplasty in different types of nasal septal deformities: our experience with NOSE evaluation

16. Aziz T, Biron VL, Ansari K, Flores-Mir C. Measurement tools for the diagnosis of nasal septal deviation: a systematic review. J Otolaryngol Head Neck Surg. 2014;43(1): 11. DOI: 10.1186/1916-0216-43-11.

17. Dell'Aversana Orabona G, Romano A, Abbate V, Salzano G, Piombino P, Farina F, et al. Effectiveness of endoscopic septoplasty in different types of nasal septal deformities: our experience with NOSE evaluation. Acta Otorhinolaryngol Ital. 2018;38(4):323-30. DOI: 10.14639/0392-100X-1067. 